\title{
Evaluation of the corpus callosum shape in patients with obstructive sleep apnea
}

\author{
Aygul Gunes $^{1} \cdot$ Deniz Sigirli $^{2} \cdot$ Ilker Ercan $^{2} \cdot$ Senem Turan Ozdemir ${ }^{3} \cdot$ Yavuz Durmus $^{4} \cdot$ Tekin Yildiz $^{5}$ (i)
}

Received: 28 April 2021 / Revised: 23 September 2021 / Accepted: 28 September 2021 / Published online: 19 October 2021

(c) The Author(s), under exclusive licence to Springer Nature Switzerland AG 2021

\begin{abstract}
Background The aim of this study was to examine whether or not there was a difference in corpus callosum shape between patients with mild to moderate and severe obstructive sleep apnea (OSA) compared with patients who have simple snoring. Methods The landmark coordinate data was obtained from the midsagittal magnetic resonance imaging (MRI) images of 70 patients who underwent polysomnography. For comparisons, mild and moderate OSA groups were combined and analyses were performed on three groups; simple snoring/control group, mild or moderate OSA group, and severe OSA group.

Results The corpus callosum shape of controls was significantly different from that of the severe OSA group. The most prominent deformities were observed in the genu and rostral body of the corpus callosum for the patients with severe OSA. No significant difference was found between mild/moderate OSA group and simple snoring group in terms of global corpus callosum shape.

Conclusion The data demonstrated that severe OSA patients have structural changes in the corpus callosum and deformities may vary as the severity of disease changes
\end{abstract}

Keywords Obstructive sleep apnea $\cdot$ Corpus callosum $\cdot$ Magnetic resonance imaging $\cdot$ Statistical shape analysis

\section{Introduction}

Obstructive sleep apnea (OSA) is a sleep disorder characterized by recurrent episodes of partial or complete obstruction of the upper airways leading to subsequent paroxysmal nocturnal hypoxia and intermittent arousals during sleep and

This study has not been presented at a conference or congress

Tekin Yildiz

drtekinyildiz@gmail.com

1 Department of Neurology, Bursa Yuksek Ihtisas Education and Training Hospital, University of Health Sciences, Bursa, Turkey

2 Department of Biostatistics, Faculty of Medicine, Uludag University, Bursa, Turkey

3 Department of Anatomy, Faculty of Medicine, Uludag University, Bursa, Turkey

4 Department of Radiology, Bursa Yuksek Ihtisas Education and Training Hospital, University of Health Sciences, Bursa, Turkey

5 Sureyyapasa Chest Diseases and Thoracic Surgery Training and Research Hospital, University of Health Sciences, Istanbul, Turkey excessive daytime sleepiness. It has a complex physiopathology, and the roles of contributing factors vary between patients with OSA [1, 2]. In the population, the prevalence of OSA with the experience of excessive daytime sleepiness is reported to be 2 to $5 \%$ in adult women and 3 to $7 \%$ in adult men [3]. The main tool for diagnosing OSA is the apnea-hypopnea index (AHI) which is calculated as the total number of apneas and hypopneas divided by the total sleep time, recorded during polysomnography (PSG). According to the American Academy of Sleep Medicine guidelines, PSG is considered to be the gold standard for diagnosing OSA [4].

Upper airway obstruction develops as a result of the interaction between many anatomical and physiological disorders. However, the main features are small pharyngeal lumen and transmural pressure [5]. The pathophysiology underlying OSA is attributable to anatomical, local, neurological, and vascular elements as well as familial predisposition. Neurological factors currently reported in some patients with OSA include abnormal respiratory control and decreased medullary respiratory neuron output as a result of autonomic activation [6]. Cognitive functions, including attention and alertness, working memory, visuospatial/structural abilities, 
and executive functions, are impaired in patients with OSA [7].

Many neuro-imaging studies have been conducted to reveal the structural changes in the brain in OSA, the pathophysiology of which has not yet been fully elucidated [8, 9]. One of the common aims of studies on neurological diseases is to examine anatomic and functional changes of the brain and to evaluate their relationship with the disruption in cognitive processes. Regional brain deformations are commonly seen with many neurological diseases. The corpus callosum (CC), which is a white matter tract that enables communication between the left and right hemispheres, is one of the most affected brain regions, and it is also known that global-local processes are affected due to structural changes in the CC $[10,11]$. The structural changes in CC, related to different neurological disorders, are usually evaluated with volumetric studies. However, it is also important to reveal regional shape deformities rather than volume differences alone. By Procrustes-based geometric morphometrics, the shape of an object is obtained which is invariant under scaling, rotation, and translation. The resulting Procrustes shape coordinates are used in statistical analysis [12]. One can obtain the same volume from different objects that have different shapes, so the shape analysis method is useful for searching sub-regional changes in different cortical and subcortical brain areas.

In the present study, we aimed to examine whether or not there were shape differences in the $\mathrm{CC}$ of patients with mild to moderate and severe OSA compared with simple snoring adults. Accordingly, we analyzed the deformities in the subregions of the CC using Witelson segmentation.

\section{Materials and methods}

\section{Patient selection}

A total of 1630 patients with OSA were screened during the study period. Since our hypothesis was to investigate changes in the CC that may be caused by the severity of OSA, 1560 patients with comorbidities were excluded from the study. A total of 70 patients who had no comorbidity were included in the study. This study was performed using the mid-sagittal magnetic resonance imaging (MRI) scans of 70 patients who underwent polysomnography in the sleep laboratory of the sleep clinic with a suspicion for OSA, between January 2017 and December 2019. Age, sex, body mass index (BMI), apnea-hypopnea index (AHI), smoking, and alcohol consumption habits of the patients were recorded retrospectively. Patient groups were constructed based on the AHI as follows: $\mathrm{AHI}<5 / \mathrm{h}$ was classified as simple snoring/control group, $5 / \mathrm{h} \leq \mathrm{AHI}<15 / \mathrm{h}$ was classified as mild OSA, $15 / \mathrm{h} \leq \mathrm{AHI}<30 / \mathrm{h}$ was classified as moderate OSA, and AHI $\geq 30 / \mathrm{h}$ was classified as severe OSA [1]. In comparisons, mild and moderate OSA groups were combined, and analyses were performed on three groups: simple snoring/control group $(n=23)$, mild to moderate OSA group $(n=24)$, and severe OSA group $(n=23)$. Patients who had neurological diseases, were older than 60 years of age, or were younger than 26 years of age were excluded from the study. We aimed to make patient groups and control group as homogeneous as possible in terms of age and gender. All procedures performed in studies involving human participants were in accordance with the ethical standards of the institutional and/or national research committee (Bursa Uludag University Faculty of Medicine Clinical Studies Ethic Committee, 2019-20/5) and with the 1964 Helsinki declaration and its later amendments or comparable ethical standards. Informed consent was received from all participants.

\section{Magnetic resonance imaging}

MRIs were performed in a 1.5-T Magnet (Magnetom Vision Plus, Siemens Medical Solutions, Erlangen, Germany) with a standard head coil. The images were obtained using a three-dimensional magnetization prepared-rapid acquisition gradient echo (3D MP-RAGE) sequences with the following parameters: TR/TE/TI/flip angle $=10 / 4 / 300 / 10^{\circ}, 250 \mathrm{FOV}$, 1.25 -mm slice, $192 \times 256$ matrix, $1 \mathrm{Nex}$. The MRI sections with good gray/white matter contrast were selected.

\section{Landmark acquisition}

The sagittal planes were evaluated by the radiologist, and the mid-sagittal section, which demonstrates the cerebral aqueduct, CC, and superior colliculus most clearly was manually selected. To align the MRI images of all of the subjects at a standard position, the commissure line from anterior to posterior and inter hemispheric fissure was used. Statistical shape analysis method has been implied by using 16 homologous landmarks, which were used in our previous studies [13-15]. The CC was divided into seven subdivisions based on the Witelson framework [16]. The landmarks in each subdivision of CC determined according to Witelson are shown on the MRI image of a control subject (Fig. 1). The landmarks were marked by using TPSDIG 2.31 software [17].

The landmark reliability was evaluated with the intrarater reliability coefficient which was calculated with a two-facet crossed design (landmark pairs-by-rater-by-subject) [18]. The landmarks were marked for all images by an investigator. To obtain a $\mathrm{G}$ reliability coefficient, after 1 month, 15 images were randomly selected and the same investigator marked the landmarks on these images. The $\mathrm{G}$ coefficient calculated showed strong repeatability for 


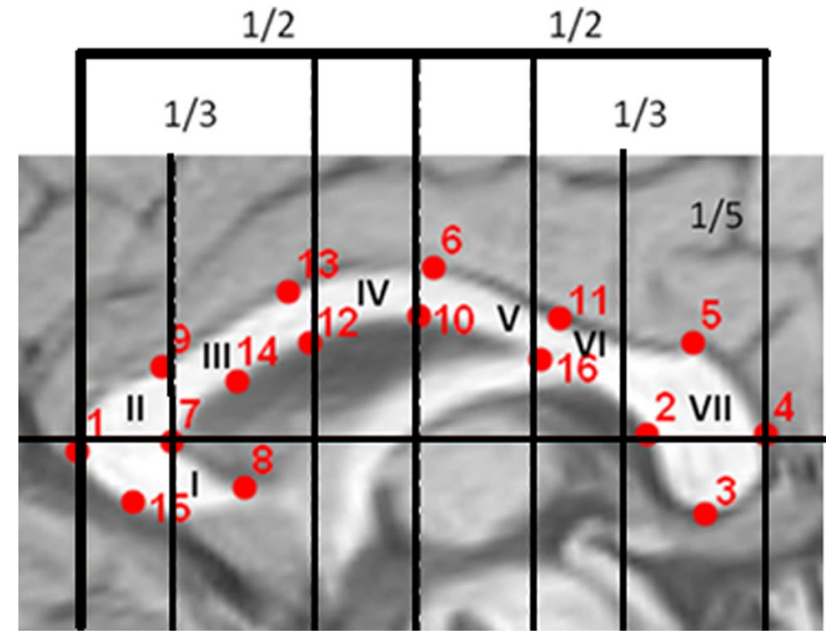

Fig. 1 T1-weighted mid-sagittal slice demonstrating the CC landmarks and Witelson subdivisions (I: rostrum; II: genu; III: rostral body; IV: anterior midbody of corpus callosum; V: posterior midbody of corpus callosum; VI: isthmus; VII: splenium)

subjects $(G=0.98)$. Landmark reliability calculations were performed from the following link: http://biostat.home. uludag.edu.tr/landmark_reliability/G_coefficient.html.

\section{Statistical analysis}

The landmark coordinate data were obtained with the generalized Procrustes analysis. The variance-covariance matrices were not homogeneous according to the Box-M test ( $p=0.022$ for simple snoring and mild/moderate groups and $p<0.001$ for simple snoring and severe groups). Accordingly, the James FJ test based on a re-sampling procedure was used to compare the shapes $[19,20]$. The differences between CC shapes of OSA and simple snoring patients are visualized using the thin-plate spline (TPS) function and deformation grids, to show the actual spatial changes [21].
By performing TPS analysis, deformities are demonstrated by the landmarks exhibiting the enlargements or reductions $[21,22]$. When the data were normally distributed, one-way analysis of variance was used; when not normally distributed, Kruskal-Wallis test was used to compare the variables between the groups. Post hoc comparisons were performed with Bonferroni test if significant differences were obtained after ANOVA. Pearson chi-square and Fisher-Freeman-Halton tests were performed to compare the categorical variables between the groups. R 4.0.0, PAST 3.20, and NCSS 7.1.5 software were used for statistical analysis.

\section{Results}

Demographic information about patients with OSA and controls are given in Table 1. When OSA and control groups were compared, no significant difference was found in terms of age and gender. There were significant differences between the three groups in terms of BMI $(p<0.0001)$.

\section{Comparison of simple snoring group and mild/ moderate OSA group}

There was no statistically significant difference between the simple snoring group and mild/moderate group in terms of CC shape ( $p=0.164)$. According to TPS graphs, very small deformities were observed in the genu, especially in the area between the landmarks 1, 7, and 9 (Fig. 2).

\section{Comparison of simple snoring group and severe OSA group}

There was a significant difference between the CC shape of the simple snorers and the $\mathrm{CC}$ shape of the patients with

Table 1 Demographic and clinical details of OSA patients and controls

\begin{tabular}{|c|c|c|c|c|c|}
\hline & & Controls (simple snoring) $(n=23)$ & Mild or moderate OSA $(n=24)$ & Severe OSA $(n=23)$ & $p$-value \\
\hline $\operatorname{Age}^{*}$ (years) & & $43.4 \pm 10.0(26-60)$ & $44.3 \pm 8.0(26-58)$ & $48.1 \pm 8.1(28-60)$ & 0.163 \\
\hline \multirow[t]{2}{*}{$\operatorname{Sex}^{\#}$} & Male & $11(48)$ & $12(50)$ & $11(48)$ & 0.985 \\
\hline & Female & $12(52)$ & $12(50)$ & $12(52)$ & \\
\hline $\mathrm{AHI}^{\&}$ & & $1.0(0.0-4.7)$ & $14.1(5.2-27.0)$ & $56.2(30.3 .-101.9)$ & $<0.001$ \\
\hline BMI & & $27.0 \pm 4.6^{1}(14.0-35.3)$ & $29.6 \pm 4.0^{2}(22.9-39.3)$ & $34.3 \pm 6.3^{3}(25.1-51.4)$ & $<0.001$ \\
\hline Smoker ${ }^{\#}$ & & $14(61)$ & $14(58)$ & $11(48)$ & 0.639 \\
\hline Alcohol user ${ }^{\#}$ & & $2(9)$ & $2(8)$ & $1(4)$ & 1.000 \\
\hline
\end{tabular}

Data given as ${ }^{*}$ mean \pm standard deviation (min-max), ${ }^{\&}$ median $(\min -\max )$ or ${ }^{\#} \mathrm{n}(\%)$

$A H I$, Apnea hypopnea index

${ }^{1}$ Significantly different from severe OSA $(p<0.001),{ }^{2}$ Significantly different from severe OSA $(p<0.007)$, ${ }^{3}$ Significantly different from mild or moderate OSA $(p=0.007)$ and from controls $(p<0.001)$ 
Fig. 2 A thin-plate spline demonstrating the average $\mathrm{CC}$ shapes deformation from simple snorers to mild/moderate patients with OSA. The expansion factors at the landmarks are shown with colors.

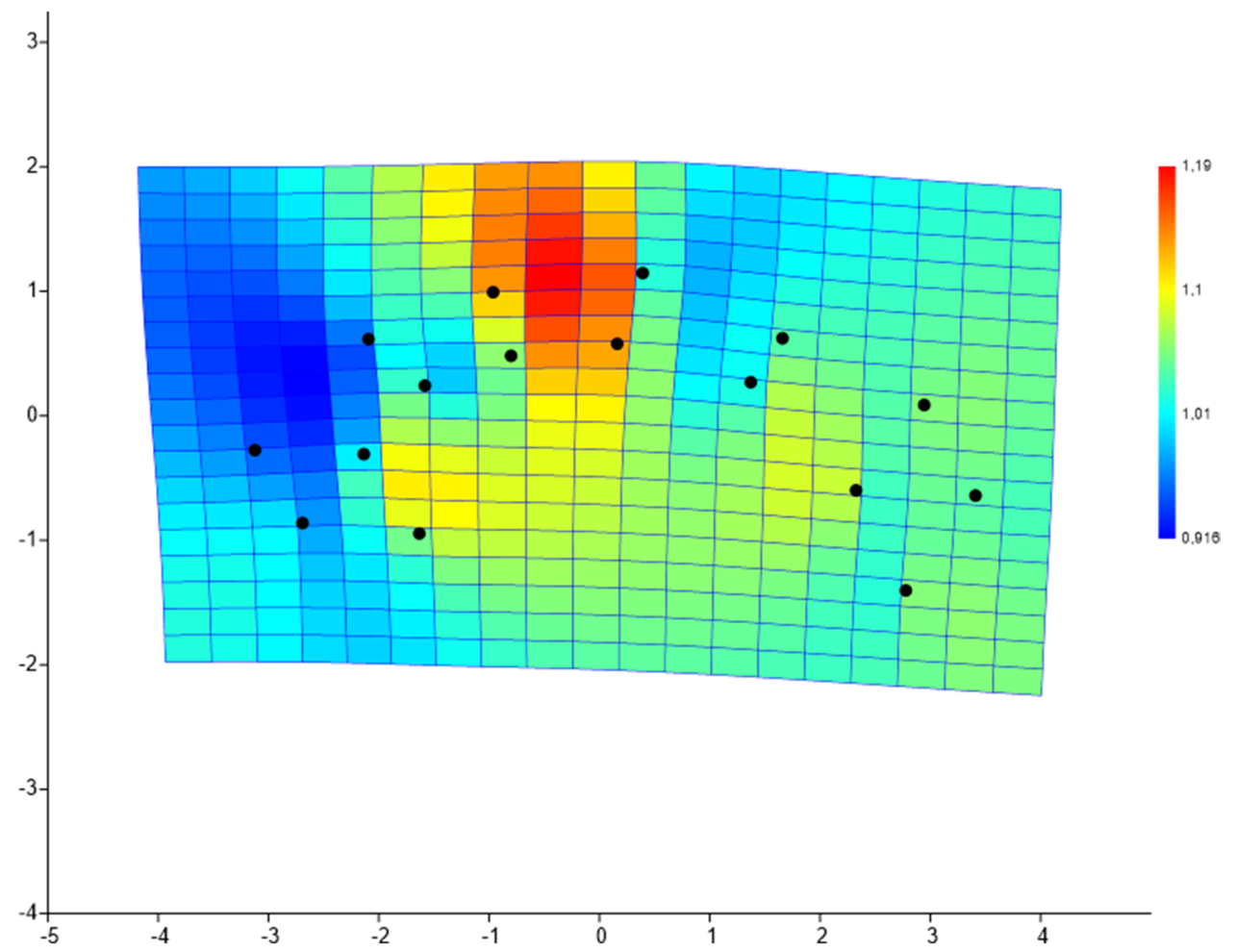

[23]. The anterior-most callosal region has the highest ratio of unmyelinated fibers. The thin fibers increase in the posterior pole of the $\mathrm{CC}$ and decrease towards the posterior midbody, while the coarse fibers are denser in the midbody of the CC [4, 23]. Histological differences also lead to functional differences, as unmyelinated thin fibers which have lower conduction velocities integrate higher-order prefrontal cortical functions while myelinated thick fibers in the midbody of CC which have higher conduction velocities integrate motor and sensory functions [24]. In the present study, we observed minimal shape deformities in the CC of patients with OSA and the most prominent deformities were in the genu of the $\mathrm{CC}$, where thin fibers are denser, both for mild to moderate OSA and for severe OSA. Our results are consistent with the study of Zhang et al. [9]. In their study, using DTI-based fiber tractography, they showed that the affected CC subregions in OSA were located in the anterior rather than posterior part and emphasized that the earlier myelination and distinctive fiber composition of the anterior CC might cause it to be more affected by OSA [9]. Kumar et al. also reported significantly reduced global brain mean diffusivity values and reported that those changes were localized in various brain regions including anterior corpus callosum in patients with OSA compared to controls, by using the diffusion tensor imaging (DTI) based mean diffusivity (MD) procedure [25]. Using DTI method, Macey et al. demonstrated that multiple regions of lower fractional anisotropy appeared within white matter in the OSA group, including fibers of the anterior $\mathrm{CC}$, along with the other 
Fig. 3 A thin-plate spline demonstrating the average $\mathrm{CC}$ shapes deformation from simple snorers to patients with severe OSA. The expansion factors at the landmarks are shown with colors.

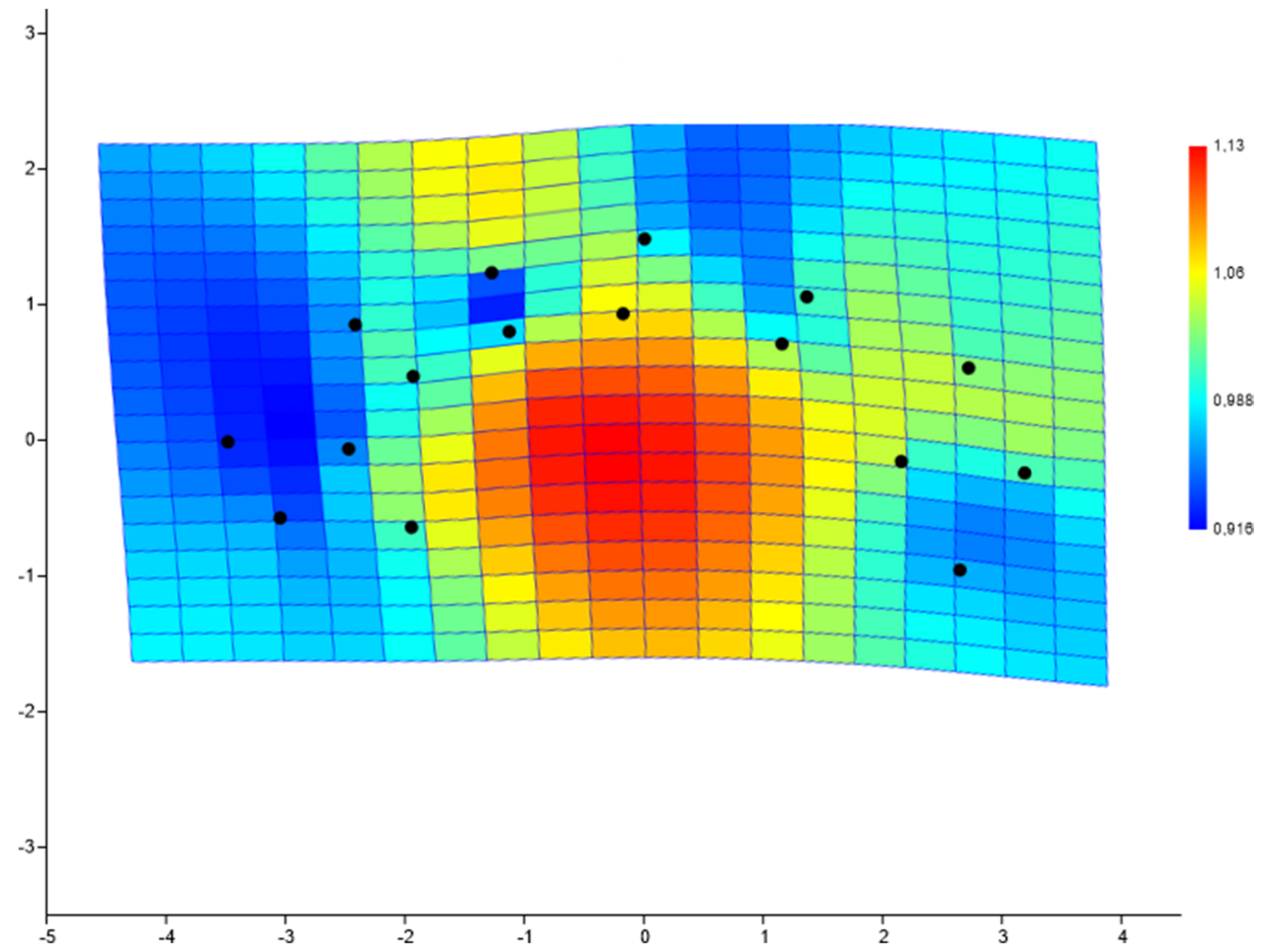

brain regions [26]. Baril et al. compared the OSA groups with DTI metrics and showed that patients with moderate to severe OSA had lower axial diffusivity in the CC compared with controls [27].

Fiber tracts from different segments of the CC provide interhemispheric connection between specific cortical regions, which have different functions. While the anteriormost point of CC links homologous prefrontal cortical regions; premotor, supplementary motor, parietal, temporal, and occipital cortices are linked from rostral to caudal regions, respectively [24]. The genu, in which we detected deformity in patients with both mild to moderate and severe OSA, contain fibers connecting the prefrontal cortices [28, 29]. The population maps of the callosal connections indicated that the parts of the dorsal prefrontal cortex and the ventral prefrontal cortex both project fibers through the genu [24]. The prefrontal cortex manages processes such as regulation of attention, differentiating among conflicting thoughts, planning, future consequences of current actions, and impulse control which is defined as executive functions. Several studies performed on animals and humans showed that the premotor cortex also participates in spatial attention and working memory, movement planning, and execution [30, 31]. Attention, vigilance, and prospective memory are processes with multiple stages and are guided by the prefrontal cortex together with the involvement of premotor and supplementary motor cortices. Cortical cells in the sensory and prefrontal region initiate movement by focusing attention and creating working memory for a motor task. Outputs from these areas are projected to the supplementary motor and the premotor cortex [32]. Cognitive impairment is common in patients with OSA, including sustained attention and vigilance, working memory, long-term episodic memory, visuospatial/constructional abilities, and executive functions $[9,26,33,34]$. This information explains the deformities that we found in the genu region connecting the prefrontal cortex in patients with mild to moderate OSA, and in the genu region as well as in the rostral body region connecting the supplementary motor and premotor cortex in patients with severe OSA. It can be emphasized that the deformities we found may be related to the mentioned cognitive dysfunctions. Also it can be speculated that deformities may move on from rostral to caudal as the disease progresses.

The results of this study show that there are global structural changes in CC when compared between simple snorers and patients with severe OSA, but not patients with mild to moderate OSA. However, according to current data, there are studies reporting that there may be a relationship between BMI and both OSA severity and CC abnormalities [35-37]. In our study, BMI values were higher in the severe OSA group in which we found CC abnormalities. Since the shape change in CC cannot be attributed to OSA alone, and even the metabolic syndrome and its outcomes may affect the CC shape, it would be appropriate to study groups that are homogeneous in terms of BMI in future studies. Nevertheless, the deformity findings that we detected more intensely in the genu and rostral body regions may 
provide important evidence that the prefrontal, premotor, and additional motor cortices are affected by OSA.

\section{Declarations}

Ethical approval All procedures performed in studies involving human participants were in accordance with the ethical standards of the institutional and/or national research committee (Bursa Uludag University Faculty of Medicine Clinical Studies Ethic Committee, 2019-20/5) and with the 1964 Helsinki declaration and its later amendments or comparable ethical standards.

Informed consent Informed consent was obtained from all individual. participants included in the study.

Conflict of interest The authors declare no competing interests.

\section{References}

1. Zhan X, Fang F, Wu C, Pinto JM, Wei Y (2018) A Retrospective study to compare the use of the mean apnea-hypopnea duration and the apnea-hypopnea index with blood oxygenation and sleep patterns in patients with obstructive sleep apnea diagnosed by polysomnography. Med SciMonit 24:1887-1893

2. American Academy of Sleep Medicine (2005) ICSD-2: The International Classification of Sleep Disorders. Diagnostic and Coding Manual, 2nd edn. AASM, Westchester

3. Punjabi NM (2008) The epidemiology of adult obstructive sleep apnea. Proc Am Thorac Soc 5(2):136-143

4. Rosen IM, Kirsch DB, Chervin RD, Carden KA, Ramar K, Aurora RN et al (2017) Clinical use of a home sleep apnea test: an American Academy of Sleep Medicine Position Statement. J Clin Sleep Med 13(10):1205-1207

5. Schwab RJ, Goldberg AN, Pack AL (1998) Sleep apnea syndromes. In: Fishman AP (ed) Fishman's Pulmonary Diseases and Disorders. McGraw Hill Book Company, New York, pp 1617-1637

6. Bradley W, Daroff RB, Fenichel GM, and Jankovic J (2008) Neurology in clinical practise, Fifth Edition, 1947-2010

7. Bilyukov RG, Nikolov MS, Pencheva VP, Petrova DS, Georgiev OB, Mondeshki TL, Milanova VK (2018) Cognitive impairment and affective disorders in patients with obstructive sleep apnea syndrome. Front Psychiatry 9:357

8. Yaouhi K, Bertran F, Clochon P, Mézenge F, Denise P, Foret J, Eustache F, Desgranges B (2009) A combined neuropsychological and brain imaging study of obstructive sleep apnea. J Sleep Res $18: 36-48$

9. Zhang B, Zhu DM, Zhao W, Zhang Y, Yang Y, Zhang C, Zhu J, Yu Y (2019) Selective microstructural integrity impairments of the anterior corpus callosum are associated with cognitive deficits in obstructive sleep apnea. Brain Behav 9(12):e01482

10. Schulte T, Müller-Oehring EM (2010) Contribution of callosal connections to the interhemispheric integration of visuomotor and cognitive processes. Neuropsychol Rev 20(2):174-190. https:// doi.org/10.1007/s11065-010-9130-1

11. Rostampour M, Noori K, Heidari M, Fadaei R, Tahmasian M, Khazaie H, Zarei M (2020) White matter alterations in patients with obstructive sleep apnea: a systematic review of diffusion MRI studies. Sleep Med 75:236-245
12. Daboul A, Ivanovska T, Bulow R, Biffar R, Cardini A (2018) Procrustes-based geometric morphometrics on MRI images: an example of inter-operator bias in 3D landmarks and its impact on big datasets. PLoS ONE 13(5):e0197675. https://doi.org/10.1371/ journal.pone.0197675

13. Ozdemir ST, Ercan I, Sevinc O, Guney I, Ocakoglu G, Aslan E, Barut C (2007) Statistical shape analysis of differences in the shape of the corpus callosum between genders. Anat Rec 290(7):825-830. https://doi.org/10.1002/ar.20558

14. Sigirli D, Ercan I, Ozdemir ST, Taskapilioglu O, Hakyemez B, Turan OF (2012) Shape analysis of the corpus callosum and the cerebellum in female MS patients with different clinical phenotypes. Anat Rec Adv Integr Anat Evol Biol 2957:1202-1211

15. Sigirli D, Gunes A, Ozdemir ST, Ercan I, Durmus Y, ErdemliGursel B (2020) Statistical shape analysis of corpus callosum in restless leg syndrome. Neurol Res 42(9):760-766. https://doi.org/ $10.1080 / 01616412.2020 .1773631$

16. Witelson SF (1989) Hand and sex differences in the isthmus and genu of the human corpus callosum. Brain 112(3):799-835

17. Rohlf FJ (2006) tpsDig, digitize landmarks and outlines, version 217. Department of Ecology and Evolution, State University of at Stony Brook, New York

18. Dimitrov D (2006) Reliability. In: Erford BT (ed) Assessment for Counselors. Houghton-Mifflin/Lahaska Press, Boston, pp 99-122

19. Dryden IL, Mardia KV (2016) Statistical shape analysis, with applications in R (Second Edition). Wiley. Chapter 9, pp 185-212

20. Dryden IL (2018) shapes package. R Foundation for Statistical Computing. Vienna: Austria

21. Bookstein FL (1992) Morphometric tools for landmark data. Cambridge University Press

22. Hammer $\emptyset$, Harper DAT, Ryan PD (2001) PAST: paleontological statistics software package for education and data analysis. Palaeontol Electron 4(1):9

23. Aboitiz F, Scheibel AB, Fisher RS, Zaidel E (1992) Fiber composition of the human corpus callosum. Brain Res 598:143-153

24. Gilliam M, Stockman M, Malek M, Sharp W, Greenstein D, Lalonde F, Clasen L, Giedd J, Rapoport J, Shaw P (2011) Developmental trajectories of the corpus callosum in attention-deficit/ hyperactivity disorder. Biol Psychiatry 69(9):839-846

25. Kumar R, Chavez AS, Macey PM, Woo MA, Yan-Go FL, Harper RM (2012) Altered global and regional brain mean diffusivity in patients with obstructive sleep apnea. J Neurosci Res 90(10):2043-2052. https://doi.org/10.1002/jnr.23083

26. Macey PM, Kumar R, Woo MA, Valladares EM, Yan-Go FL, Harper RM (2008) Brain structural changes in obstructive sleep apnea. Sleep 31(7):967-977

27. Baril AA, Gagnon K, Descoteaux M, Bedetti C, Chami S, Sanchez E, Montplaisir J, De Beaumont L, Gilbert D, Poirier J, Pelleieux S, Osorio RS, Carrier J, Gosselin N (2020) Cerebral white matter diffusion properties and free-water with obstructive sleep apnea severity in older adults. Hum Brain Mapp 41(10):2686-2701. https://doi.org/10.1002/hbm.24971

28. de MeloMussi AC, da Luz de Oliveira EP (2019) Ventricular anatomy. In: Comprehensive Overview of Modern Surgical Approaches to Intrinsic Brain Tumors. Chapter 5 pp:107-118

29. Zhang B, Zhu DM, Zhao W, Zhang Y, Yang Y, Zhang C, Zhu J, Yu Y (2019) Selective microstructural integrity impairments of the anterior corpus callosum are associated with cognitive deficits in obstructive sleep apnea. Brain Behav 9(12):e01482

30. Baleydier C, Achache P, Froment JC (1997) Neurofilament architecture of superior and mesial premotor cortex in the human brain. NeuroReport 8:1691-1696

31. Simon SR, Meunier M, Piettre L, Berardi AM, Segebarth CM, Boussaoud D (2002) Spatial attention and memory versus motor preparation: premotor cortex involvement as revealed by fMRI. J Neurophysiol 88(4):2047-2057 
32. Feher J (2012) Balance and Control of Movement. Editor(s): Joseph Feher, Quantitative Human Physiology (Second Edition), Academic Press, pp 409-421

33. Findley LJ, Barth JT, Powers DC, Wilhoit SC, Boyd DG, Suratt PM (1986) Cognitive impairment in patients with obstructive sleep apnea and associated hypoxemia. Chest 90:686-690

34. Solorio C, White D, Piccirillo J, Duntley SP, Uhles ML (2002) Learning, memory, and executive control in individuals with obstructive sleep apnea syndrome. J Clin Exp Neuropsychol 24:93-100

35. Xu J, Li Y, Lin H, Sinha R, Potenza MN (2013) Body mass index correlates negatively with white matter integrity in the fornix and corpus callosum: a diffusion tensor imaging study. Hum Brain Mapp 34(5):1044-1052. https://doi.org/10.1002/hbm.21491

36. Repple J, Opel N, Meinert S, Redlich R, Hahn T, Winter NR, Kaehler C, Emden D, Leenings R, Grotegerd D, Zaremba D, Bürger C, Förster K, Dohm K, Enneking V, Leehr EJ, Böhnlein J, Karliczek G, Heindel W, Kugel H, Bauer J, Arolt V, Dannlowski $\mathrm{U}$ (2018) Elevated body-mass index is associated with reduced white matter integrity in two large independent cohorts. Psychoneuroendocrinology 91:179-185. https://doi.org/10.1016/j.psyne uen.2018.03.007

37. Papageorgiou I, Astrakas LG, Xydis V, Alexiou GA, Bargiotas P, Tzarouchi L, Zikou AK, Kiortsis DN, Argyropoulou MI (2017) Abnormalities of brain neural circuits related to obesity: a diffusion tensor imaging study. Magn Reson Imaging 37:116-121. https://doi.org/10.1016/j.mri.2016.11.018

Publisher's note Springer Nature remains neutral with regard to jurisdictional claims in published maps and institutional affiliations.

Mandatory disclosure and commemoration Dr. Yavuz DURMUS evaluated MR examinations of all patients and control group in this study. Unfortunately, he died on 28 December2020, due to COVID-19. We bow respectfully before his memory. 